\title{
Vocal Cord Paralysis
}

National Cancer Institute

\section{Source}

National Cancer Institute. Vocal Cord Paralysis. NCI Thesaurus. Code C47814.

Paralysis of the muscles of one or both vocal cords. Signs and symptoms include hoarseness, weak voice, dyspnea, and coughing. Affected individuals are at risk for aspiration pneumonia. 